Monsieur Christophe Defeuilley Monsieur Philippe Quirion

\title{
Les déchets d'emballages ménagers : une analyse économique des politiques allemande et française
}

In: Economie et statistique, №290, 1995. pp. 69-79.

Citer ce document / Cite this document :

Defeuilley Christophe, Quirion Philippe. Les déchets d'emballages ménagers : une analyse économique des politiques allemande et française. In: Economie et statistique, N²90, 1995. pp. 69-79.

doi : $10.3406 /$ estat. 1995.6020

http://www.persee.fr/web/revues/home/prescript/article/estat_0336-1454_1995_num_290_1_6020 


\section{Résumé}

place accordée à l'incinération et le degré d'implication des collectivités locales.

Les déchets d'emballages ménagers : une analyse économique des politiques allemande et française La gestion des déchets d'emballages ménagers connaît des évolutions profondes depuis 1991 en Allemagne et 1992 en France. Dans les deux pays, c'est un organisme mis en place par les industriels qui finance la collecte sélective des déchets grâce à une contribution perçue sur les produits emballés. Mais il existe trois différences marquées entre les deux systèmes : l'importance des mesures limitant « à la source » la production d'emballages, la place accordée à l'incinération et le degré d'implicitation des collectivités locales.

La théorie économique peut contribuer à éclairer les choix effectués dans chacun des deux pays en matière de collecte sélective et de production d'emballages. Comparé au système français, celui mis en place en Allemagne fait payer davantage aux producteurs d'emballages les coûts externes induits par les déchets ménagers, notamment la tieux à court terme. En comparaison, la politique française pollution. La mise en place du dispositif allemand s'est heur- apparaît plus prudente, même si la collecte sélective ne tée à d'importantes difficultés du fait d'objectifs très ambi- semble se développer que très progressivement.

\section{Abstract}

Household Packaging Waste: An Economic Analysis of the German and French Policies

The management of household packaging waste has developed a great deal in Germany since 1991 and in France since 1992. In both countries, an organization has been set up by manufacturers to finance selective waste collection from a levy paid on packaged products. This said, there are three marked differences between the two systems: the extent of the measures limiting packaging production "at source", the weight of incineration in waste disposal, and the amount of local authority involvement. Economic theory is able to throw some light on the choices made by each of the two countries as regards selective collection and packaging production. When compared with the French system, the German scheme is found to make packaging producers pay a higher proportion of the external costs resulting from household waste and pollution in particular. The German system has experienced substantial problems in its setting up due to extremely ambitious short-term goals. The French policy is comparatively more prudent, although selective collection seems to be developing but very gradually.

\section{Zusammenfassung}

Der Verpackungsmùll der Privathaushalte: eine wirtschaftliche Analyse der deutschen und der franzôsischen Politik

Bei der Entsorgung des Verpackungsmùlls der Privathaushalte gehen in Deutschland seit 1991 und in Frankreich seit 1992 tiefgreifende Entwicklungen vonstatten. In beiden Làndern finanziert eine von den Industrieuntemehmen gegrundete Einrichtung das getrennte Einsammeln des Mùlls mit Hilfe einer Abgabe, die auf die verpackten Erzeugnisse

erhoben wird. Zwischen den beiden Systemen bestehen jedoch drei wesentliche Unterschiede: der Umfang der MaBnahmen, die an der 'fôuelle" die+Herstellùng von Verpackungsmaterialien begrenzen, der Stellenwert, der der Verbrennung eingeràumt wird, sowie der Grad der Beteiligung der Gebietskôrperschaften.' Die Wirtschaftstheorie kann AufschluB uber die Wahl geben, die die beiden Lander im Bereich der Herstellung und der getrennten Einsammlung von Verpackungs- materialien getroffen haben. Im Vergleich zum franzô- sischen System mussen bei dem in Deutschland geschaffenen System die Hersteller von Verpackungsmaterialien verstàrkt fur die extemen Kosten aufkommen, die durch

den Hausmull, insbesondere die Verschmutzung anfallen. Aufgrund der uberaus ehrgeizigen kurzfristigen Ziele stieB die Errichtung des deutschen Systems allerdings auf erhebliche Schwierigkeiten. Im Vergleich dazu ist die franzôsische Politik vorsichtiger, auch wenn sich die getrennte Einsammlung nur sehr allmahlich durch- zusetzen scheint.

Resumen

Los desechos de los envases : un anâlisis econômico de las polfticas alemana y francesa

La gestion de los desechos de los envases expérimenta unas evoluciones profundas desde 1991 en 
Alemania y 1992 en Francia. En ambos paises, es un organismo que fundaron los industriales, el que financia la colecta sêlectiva de los desechos gracias a una contribution recaudada en los productos envasados. Pero existen très senaladas diferencias entre los dos sistemas : la importancia de las medidas que limitan al origen la production de los envases, la importancia que se le confiere a la incineration y el grado de implication de las administraciones locales.

Las ensenanzas que se deducen de la teorfa econômica permiten aclarar las orientaciones que sigue cada pais en cuanto a colecta sêlectiva y producciôn de envases. Comparado con el sistema francés, el que se puso en prâctica en Alemania ante todo les hace pagar a los productores de envases los costes externos inducidos por los desechos, en especial la contaminaciôn. Este se enfrentô sin embargo con unas dificultades que se debieron a unos objetivos muy ambiciosos a corto plazo. En comparaciôn, la polftica francesa es mucho mes prudente, aunque la colecta selectiva no parece desarrollarse sino muy paulatinamente. 


\section{Les déchets d'emballages ménagers : une analyse économique des politiques allemande et française}

Christophe Defeuilley et Philippe Quirion*
*Christophe Defeuilley fait partie du Centre international de recherche sur l'environnement et le développement (Cired), EHESS et CNRS; Philippe Quirion faisait partie de la division Marchés et stratégies d'entreprise de l'insee au moment de la rédaction de cet article ; il est actuellement au Cema (Centre d'économie industrielle de l'école des Mines de Paris.

Les auteurs remercient Michel Beaud, Laurence Bloch, Sylviane Gastaldo, Matthieu Glachant et deux lecteurs anonymes pour leurs remarques et leurs suggestions.

Les noms et dates entre parenthèses renvoient à la bibliographie en fin d'article.
La gestion des déchets d'emballages ménagers connaît des évolutions profondes depuis 1991 en Allemagne et 1992 en France. Dans les deux pays, c'est un organisme mis en place par les industriels qui finance la collecte sélective des déchets grâce à une contribution perçue sur les produits emballés. Mais il existe trois différences marquées entre les deux systèmes : l'importance des mesures limitant « à la source » la production d'emballages, la place accordée à l'incinération et le degré d'implication des collectivités locales.

La théorie économique peut contribuer à éclairer les choix effectués dans chacun des deux pays en matière de collecte sélective et de production d'emballages. Comparé au système français, celui mis en place en Allemagne fait payer davantage aux producteurs d'emballages les coûts externes induits par les déchets ménagers, notamment la pollution. La mise en place du dispositif allemand s'est heurtée à d'importantes difficultés du fait d'objectifs très ambitieux à court terme. En comparaison, la politique française apparaît plus prudente, même si la collecte sélective ne semble se développer que très progressivement.

4 ace à la montée des préoccupations environnementales liées à l'élimination des déchets et aux difficultés croissantes d'implantation des décharges et des usines d'incinération, différents instruments de régulation (normes, taxes, systèmes de consigne obligatoires, interdictions...) ont été introduits dans de nombreux pays (OCDE, 1992). Ces instruments visent à limiter le recours à l'élimination (mise en décharge et, dans certains pays, incinération) soit par la réduction «à la source » de la production d'emballages, soit par le développement d'autres modes de traitement (recyclage et parfois incinération avec récupération d'énergie). Les mécanismes instaurés récemment en Allemagne et en France pour maîtriser les déchets d'emballages répondent différemment à ces objectifs. Nous tenterons de déterminer, à la lumière de la théorie économique, la pertinence et les limites de ces deux politiques. 
1. Pour les comparaisons statistiques, on se reportera à l'encadré 1 .

2. Le décret Töpfer fixe aussi des taux de consignation obligatoire pour certains emballages de boissons, mais nous ne détaillons pas cet aspect ici.

\section{Les politiques allemande et française}

$\mathrm{L}$ a comparaison des deux politiques publiques fait apparaître un point commun, la création dans chaque pays d'une société privée finançant la collecte sélective grâce à une contribution perçue sur les produits emballés, et trois différences essentielles : l'importance des mesures de réduction de la quantité de déchets à la source, la place réservée à l'incinération et l'implication des collectivités locales (1).

\section{Le système DSD en Allemagne}

La politique publique de gestion des déchets d'emballages ménagers s'est mise en place en Allemagne un an plus tôt qu'en France, avec le décret Töpfer du 19 avril 1991. Cette réglementation avait deux objectifs : limiter la production d'emballages et développer leur recyclage, jusqu'à atteindre des taux de recyclage de $64 \%$ à $72 \%$ selon les matériaux, d'ici au $1^{\text {er juillet }}$ 1995 (DSD, 1994) (2). Les industriels allemands ont conçu un moyen original pour appliquer la réglementation. Ils ont constitué une société privée, la Duales System Deutschland $\mathrm{GmbH}$ (DSD), qui a pour mission d'organiser la collecte et le tri. Le capital de la DSD est réparti à parts égales entre les distributeurs, les fabricants d'emballages et les conditionneurs (3).
Les activités de la société sont financées par une taxe perçue sur les emballages mis en vente (4), qui donne droit à l'apposition d'un «point vert » sur ces emballages.

La DSD s'acquitte de deux rôles (cf. schéma I). D'abord, elle finance la mise en place des systèmes de collecte sélective et de tri des déchets. Développés par des opérateurs publics ou privés, ces systèmes permettent de récupérer les matériaux issus des déchets d'emballages ménagers en vue d'un recyclage ultérieur (5). La seconde tâche de la DSD consiste à faciliter la stabilisation des conditions de reprise pour les matériaux récupérés, dont les marchés sont traditionnellement caractérisés par une grande volatilité. Pour cela, des sociétés chargées d'organiser et de garantir le recyclage ont été créées, le plus souvent par les fabricants de matériaux d'emballages. Elles s'engagent par contrat avec la DSD à reprendre et à recycler les déchets triés. Jusqu'en octobre 1994 (juin 1993 pour les plastiques), cette reprise se faisait à « prix zéro », alors que les prix sur les marchés de la récupération sont traditionnellement positifs pour certains matériaux (verre, aluminium, fer/acier) et négatifs pour d'autres (plastiques).

3. On regroupe sous ce termelesproducteurs et les importateurs de produits emballés.

4. Cette contribution est perçue sur les produits aussi bien importés que nationaux, mais pas sur les produits exportés. De ce fait, elle n'a pas d'effet négatif en termes de compétitivité.

5. Les communes restent responsables de la collecte des autres déchets ménagers.

\section{Schéma I}

Le dispositif de la DSD

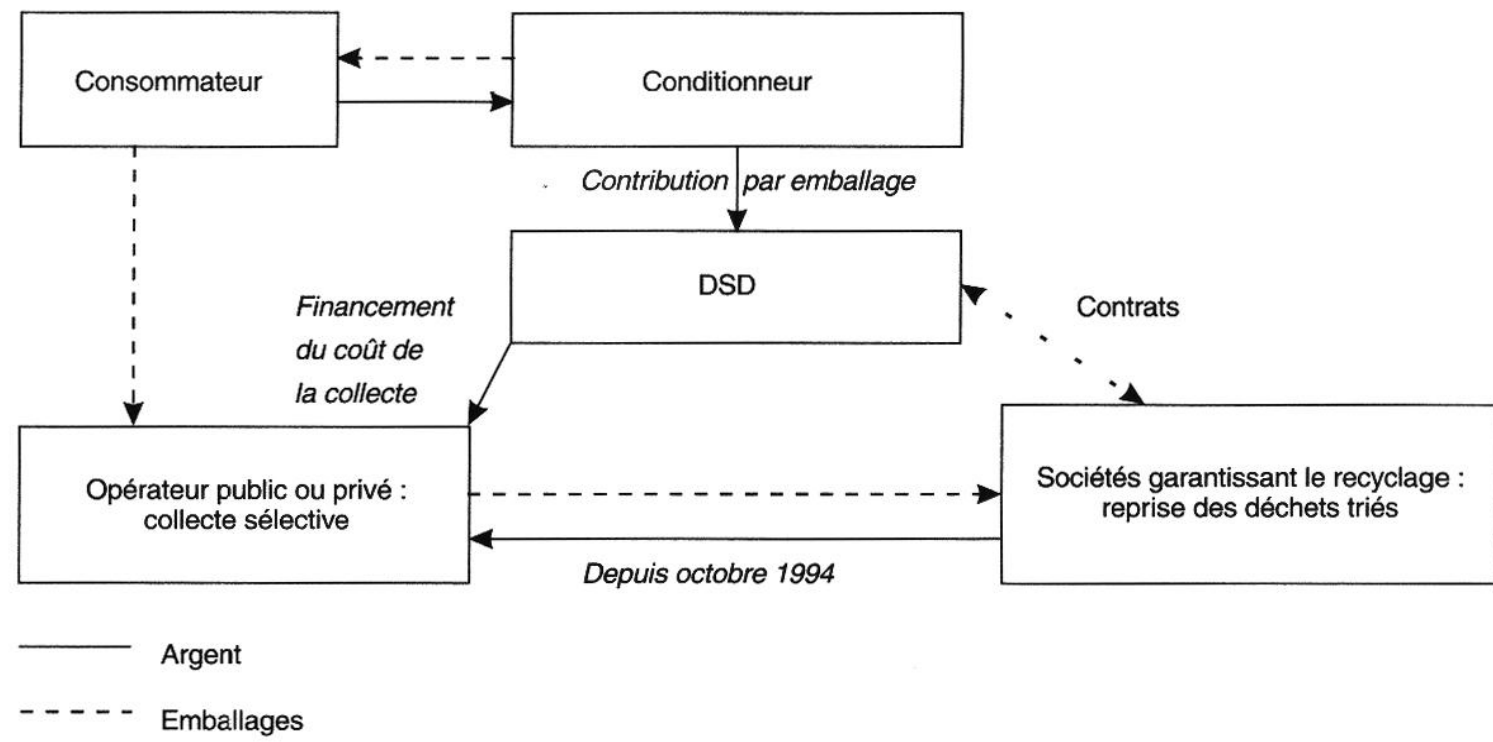


Ce système du prix zéro, favorable aux sociétés qui recyclent les emballages de la première catégorie, a été institué en compensation de la garantie de reprise et de recyclage que ces sociétés assurent (DSD, 1995).

La montée en puissance de la DSD s'est effectuée extrêmement rapidement, puisque 1,5 million de tonnes de matériaux d'emballages ont été collectées en 1992 et 4,4 millions l'année suivante (Bially, 1993). En 1993 toutefois, le dispositif allemand a affronté une double crise. La première touchait directement la firme DSD, qui ne pouvait plus financer les opérations de collecte et de tri. La cause de cette incapacité était double ; d'une part le coût de ces opérations avait été largement sous-estimé et les contributions se révélèrent donc insuffisantes, d'autre part un grand nombre de conditionneurs fraudaient en ne payant pas leur licence ou en déclarant une quantité d'emballages mis sur le marché inférieure à la réalité.

La seconde crise concernait le recyclage des plastiques. En effet, le choix d'une utilisation exclusive du recyclage comme mode de valorisation s'est soldé par l'émergence d'un écart important entre les quantités triées par les ménages (plus de 400000 tonnes dès la première année) et les capacités des installations de recyclage (entre 100000 et 250000 tonnes par an selon les estimations).

De ce fait, une partie des emballages plastiques collectés outre-Rhin s'est vu exportée à prix négatifs, c'est-à-dire avec un chèque d'accompa- gnement, pour finir en décharge. En outre, des déchets d'emballages papier-carton se sont eux aussi vu exportés à prix négatifs vers les pays limitrophes, en particulier la France.

La DSD a réagi en augmentant le montant de la contribution qu'elle perçoit sur les emballages, en changeant son mode de calcul et en renonçant au principe de reprise des matériaux récupérés à prix zéro. Depuis octobre 1993 en effet, la contribution n'est plus principalement fonction du volume de l'emballage, mais surtout de son poids et du matériau utilisé (cf. tableau 1). Le but recherché est double : accroître les rentrées financières et limiter la production des

\section{Tableau 1 \\ Barème de contribution à la DSD* (depuis octobre 1994)}

\begin{tabular}{|l|c|}
\hline \multicolumn{1}{|c|}{ Type d'emballage } & Francs/kg (1) \\
\hline Plastiques & 10,03 \\
Emballages complexes & 7,14 \\
Cartons pour boisson & 5,75 \\
Aluminium & 5,10 \\
Fer - Acier & 1,90 \\
Papier - Carton & 1,36 \\
Verre & 0,51 \\
Matériaux naturels & 0,68 \\
\hline 1. Sur la base de 1 DM pour 3,4 francs. 100 Pf. valent 1 DM. \\
\hline
\end{tabular}

*À ces contributions s'ajoute une contribution proportionnelle au volume de l'emballage (de 0,1 à 1,2 Pf.) ou à sa surface (de 0,1 à 0,9 Pf.)

Source : DSD, 1995.

\section{Encadré 1}

\section{DONNÉES STATISTIQUES}

Les comparaisons internationales en matière de déchets ménagers sont extrêmement difficiles faute d'accord sur les définitions statistiques. Les données citées ici ne le sont donc qu'à titre d'indication. On estime à 20,5 millions de tonnes la production annuelle de déchets ménagers en France (soit $360 \mathrm{~kg}$ par habitant), contre 23,5 millions de tonnes en Allema- gne (soit $368 \mathrm{~kg}$ par habitant, ancienne Allemagne de l'Ouest uniquement). Dans chaque pays, les emballages représentent environ un tiers des ordures ménagères en poids et $50 \%$ en volume. Les modes de traitement utilisés sont présentés dans le tableau ci-dessous.

\begin{tabular}{|l|c|c|c|c|c|c|}
\hline & Décharge & Incinération & Compostage & Recyclage & Autres & Total \\
\hline Allemagne & 64 & 20 & 4 & 9 & 3 & 100 \\
France & 52 & 38 & 6 & 4 & 0 & 100 \\
\hline
\end{tabular}

Sources : pour l'Allemagne, calculs des auteurs d'après OCDE. Données surl'environnement 1993 et OCDE, Examen des performances environnementales: l'Allemagne, 1993 ; pour la France, Ademe (Agence de l'environnement et de la maîtrise de l'énergie). Toutes les données concernent l'année 1990. 
emballages les plus difficiles à recycler. Par ailleurs, depuis le $1^{\text {er }}$ octobre 1994, de nouveaux contrats de reprise des déchets triés ont été signés entre la DSD et les sociétés qui garantissent le recyclage, à des prix proches de ceux du marché.

Cet abandon du système du « prix zéro » vise d'une part à ce que la DSD bénéficie de la vente des matériaux qui se négocient à prix positifs (verre, métaux), et d'autre part à financer le recyclage des matières plastiques. En effet, cette dernière activité n'est actuellement pas rentable si les vieux plastiques à recycler sont achetés à prix nul.

\section{Le programme français}

Si le système français s'inspire du dispositif allemand, son élaboration a davantage mis l'accent sur la concertation avec les milieux industriels. Les pouvoirs publics ont notamment largement consulté les grandes firmes du secteur de l'emballage (BSN et Saint-Gobain), qui ont mis au point des propositions d'organisation (voir les rapports Riboud et Beffa de juin et octobre 1991) reprises, pour la plupart d'entre elles, dans la réglementation.

Le ministère de l'Environnement a fixé comme objectif la valorisation de $75 \%$ des emballages ménagers en 2002. Les options techniques qui devront être utilisées pour atteindre cet objectif ne sont pas spécifiées : cette valorisation peut être « énergétique » (incinération avec récupération d'énergie) ou « matière » (recyclage) (6). La liberté de choix revient aux collectivités locales, qui gardent la responsabilité de la collecte et du traitement des déchets ménagers.

Pour répondre à l'obligation de valoriser leurs emballages d'ici 2002, le décret d'avril 1992 laisse deux possibilités aux conditionneurs : pourvoir eux-mêmes à la gestion de leurs déchets d'emballages, soit en créant un système de consigne, soit en créant des emplacements spécifiquement destinés à recevoir les déchets d'emballages, ou adhérer à un organisme agréé par l'État. Dans leur grande majorité, les industriels ont opté pour la seconde solution en adhérant à Éco-Emballages (7). Il s'agit d'une société privée dont le capital est détenu majoritairement par les conditionneurs et, de façon minoritaire, par les distributeurs et les filières de matériaux (producteurs de matériaux, producteurs d'emballages, récupérateurs...).

6. Certes, l'arrêté d'agrément d'Éco-Emballages précise qu'il est "pris acte de l'hypothèse avancée [par Éco-Emballages] selon laquelle cette valorisation pourrait être assurée pour les trois quarts sous forme de recyclage et pour le reste par une valorisationénergétique ". Maisilne s'agitpaslàd'une obligation contractuelle.

7. Deux autres sociétés ont reçul'agrément: Adelphe secharge de la valorisation des bouteilles de verre et Cyclamed des emballages de médicaments.

\section{Schéma II \\ Le dispositif Éco-Emballages}

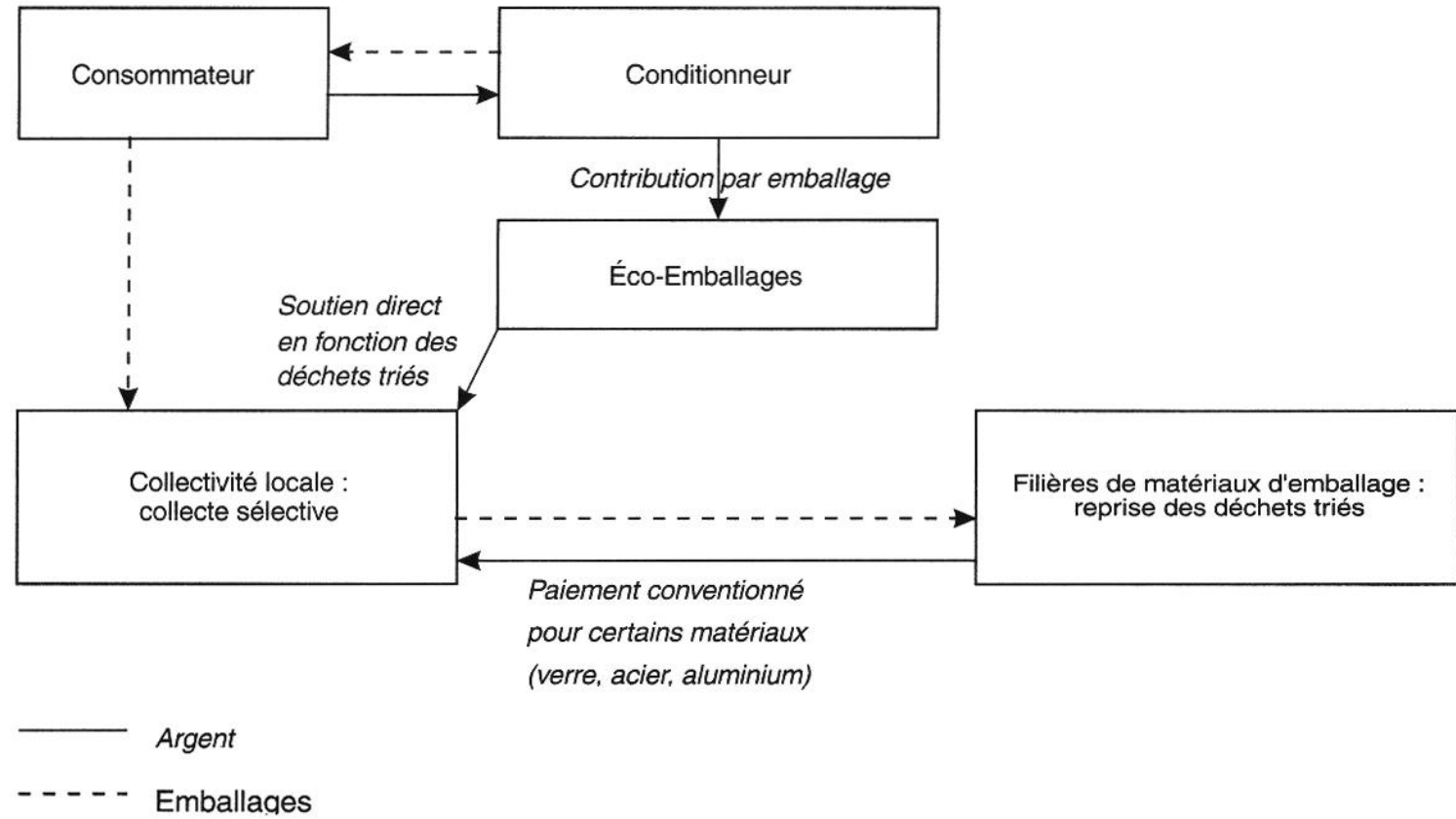


8. Éco-Emballages n'a pas pour vocation d'aider la totalité des collectivités locales. Seules seront aidées celles qui s'engagent à mettre en place des systèmes de collecte et de tri des déchets d'emballage répondant au cahier des charges défini parla sociéte.
Éco-Emballages fonctionne sur le même principe que la DSD (cf. schéma II). Les conditionneurs qui adhèrent à cette firme payent une contribution sur chaque emballage et reçoivent en échange le droit d'y apposer le même « point vert » que celui utilisé par la DSD. Le produit de cette contribution est utilisé pour soutenir financièrement les opérations de collecte sélective et de tri menées par les collectivités locales (8). La contribution est en moyenne d'un centime par emballage (cf. tableau 2), et devrait passer à trois centimes dans quelques années, après une montée en puissance du système. Contrairement à la configuration allemande, cette taxe est indifférenciée : tous les emballages, quels que soient les matériaux qui entrent dans leur composition, subissent la même contrainte. Il est toutefois prévu de la différencier à terme (Beffa, 1991), lorsque les coûts de collecte et de traitement seront mieux connus.

\section{Tableau 2}

Barème des contributions à Éco-Emballages*

\begin{tabular}{|l|c|}
\multicolumn{1}{c|}{} & En centimes \\
\hline Plus de $30001 \mathrm{~cm}^{3}$ & 10,00 \\
\hline 3001 à $30000 \mathrm{~cm}^{3}$ & 2,50 \\
\hline 201 à $3000 \mathrm{~cm}^{3}$ & 1,00 \\
\hline 151 à $200 \mathrm{~cm}^{3}$ & 0,50 \\
\hline 101 à $150 \mathrm{~cm}^{3}$ & 0,25 \\
\hline 50 à $100 \mathrm{~cm}^{3}$ & 0,10 \\
\hline Moins de $50 \mathrm{~cm}^{3}$ & $\begin{array}{c}\text { au poids } \\
\text { (plafond à } 0,10 \text { centime) }\end{array}$ \\
\hline
\end{tabular}

*Pour les emballages " corps creux rigides".

Source : Éco-Emballages, 1992, p. 50.
Éco-Emballages assure une recette minimale aux collectivités locales avec lesquelles elle signe un contrat. Cette recette minimale est issue d'un double paiement des déchets triés : un soutien direct assuré par Éco-Emballages et un prix de reprise garanti payé par les filières de matériaux chargées du recyclage (cf. tableau 3). Ces filières participent à Éco-Emballages et passent des contrats avec cette société pour garantir, matériau par matériau, les prix de reprise des déchets, pour lesquels des normes de qualité devront être respectées. L'objet de ces derniers contrats n'est pas de créer un transfert de ressources des producteurs de matériaux vers les collectivités locales, les prix garantis étant proches des prix de marché, mais de stabiliser les marchés des matières de récupération afin de pérenniser les investissements des collectivités locales.

La montée en puissance du dispositif français apparaît plus lente que celle de la DSD. Au 30 juin 1995, soit deux ans et demi après l'entrée en vigueur du décret d'avril 1992, seuls 3 millions d'habitants, soit $5 \%$ de la population, triaient leurs déchets dans le cadre de programmes de collecte sélective aidés par Éco-Emballages.

\section{Éco-Emballages et DSD : une même implication du monde industriel...}

Les systèmes allemand et français se caractérisent avant tout par le rôle central qu'y jouent des sociétés privées. Cette singularité soulève une question : pourquoi les industriels ont-ils ainsi « proposé leurs services »?

Tableau 3

Versements d'Éco-Emballages et des filières de matériaux aux collectivités locales

\begin{tabular}{|l|r|r|r|}
\hline & Soutien direct Éco-Emballages & $\begin{array}{c}\text { Prix de reprise garanti } \\
\text { par les filières }\end{array}$ & $\begin{array}{c}\text { Recette minimale } \\
\text { de la collectivité }\end{array}$ \\
\hline Plastique & 1500 & enlèvement gratuit & 1500 \\
Aluminium : & 1500 & 1000 à 1 500 & 2500 \\
- non incinéré & 500 & 500 à 1 1000 & 1000 \\
- incinéré & 300 & & \\
Fer - Acier: & 75 & 50 à 200 & 350 \\
- non incinéré & 750 & 0 à 50 & 75 \\
- incinéré & 0 à 50 & enlèvement gratuit & 750 \\
Papier - Carton & & 150 & 150 \\
Verre & & & \\
\hline
\end{tabular}

Source : Éco-Emballages, 1993, pp. 10-11. 
En acceptant de prendre en charge la traduction effective des objectifs fixés par les pouvoirs publics, l'industrie se donne les moyens de limiter le risque d'une intervention publique plus « autoritaire ». En Allemagne, cette menace était très claire : sans la mise en place de la DSD, les industriels se seraient trouvés dans l'obligation de récupérer eux-mêmes leurs déchets d'emballages, pour un coût probablement bien supérieur.

\section{... mais des principes différents}

La similitude formelle des deux systèmes ne doit pas faire oublier leurs différences, qui concernent principalement trois points. D'une part, la contribution utilisée en Allemagne est différenciée par matériau et d'un montant beaucoup plus élevé que celle qui existe en France. De ce fait, elle possède un volet incitatif, visant explicitement à limiter l'utilisation des emballages et à réorienter la production vers des matériaux plus facilement recyclables. Au contraire, la contribution française est d'un niveau faible et n'est pas différenciée, la politique française ne cherchant pas à agir sur la production de déchets.

La seconde différence importante concerne les techniques retenues pour traiter les déchets d'emballages. L'incinération avec récupération d'énergie, qualifiée de « valorisation énergétique » est considérée en France comme équivalente au recyclage. L'objectif fixé par le ministère de l'Environnement $(75 \%$ de « valorisation » en 2002) doit donc être atteint par une combinaison de ces deux modes de traitement. En Allemagne au contraire, l'objectif du ministère ( $64 \%$ à $72 \%$ selon les matériaux) doit être atteint par le seul recyclage, ce qui implique la mise en place extrêmement rapide de capacités de recyclage importantes, en particulier pour les emballages plastiques.

Enfin, la responsabilité de la collecte des déchets d'emballages est confiée à la DSD en Allemagne alors qu'elle reste du ressort des collectivités locales en France. Éco-Emballages possède donc des responsabilités plus limitées que la firme allemande.

Ceci conduit à la définition d'un graphique organisationnel plus simple en France : l'unité du système de collecte est respectée, alors qu'en Allemagne deux systèmes coexistent, l'un pour les emballages, l'autre pour le reste des déchets ménagers. Ce dédoublement du système de collecte peut être un facteur important de renchérissement des coûts.

\section{Intérêt et limites des systèmes mis en place : l'apport de la théorie économique}

es choix effectués dans chacun des deux
pays peuvent être confrontés aux enseignements fournis par la théorie économique. Habituellement, l'économie prend en compte l'environnement grâce à la notion d'externalité (ou effet externe), définie comme une interdépendance non volontaire entre agents économiques (producteurs ou consommateurs). L'exemple d'effet externe le plus simple est celui du cours d'eau. Un agent rejette des substances polluantes et détériore ainsi la qualité de l'eau. Les nuisances que ce comportement entraîne pour les agents situés en aval ne sont pas prises en compte par le pollueur, et la quantité d'effluents rejetée sera supérieure à ce qu'elle serait à l'optimum. Si les agents concernés ne négocient pas, on se trouve en présence d'un « marché manquant », qui empêche d'aboutir à un optimum de Pareto. L'État doit alors intervenir, par exemple en taxant ou en réglementant l'émission de substances polluantes, de façon à « internaliser » l'externalité. Un tel problème ne fait intervenir que deux groupes d'agents, les «pollueurs » et les "victimes ». La situation est plus complexe en ce qui concerne'les emballages ménagers, puisqu'il existe non pas un, mais deux « marchés manquants » (cf. schéma III) et par conséquent deux types de coûts externes.

D'une part, le service de collecte assuré par les collectivités locales est facturé aux ménages à un tarif qui ne tient pas compte de leur production réelle de déchets. Pour un ménage, et plus généralement pour tout agent situé en amont (producteur d'emballages, conditionneur, distributeur), le coût marginal que représente le rejet d'un déchet est nul puisque les impôts locaux croissent en fonction de la quantité de déchets rejetée par l'ensemble des ménages de sa commune et donc en proportion négligeable de la quantité de déchets que lui-même engendre. Or, l'augmentation de la quantité de déchets accroît le coût de la collecte et du traitement, payé par les collectivités locales, et donc en définitive par les contribuables.

D'autre part, le traitement de ces déchets cause des dommages à la santé humaine et à l'environnement (pollution due à l'incinération, à la mise en décharge...). Grâce à l'instauration de réglementations plus sévères, comme la limita- 
tion de la mise en décharge, les normes d'émissions pour l'incinération..., ces dommages tendent à diminuer. Toutefois, il subsistera toujours des pollutions résiduelles, qui doivent être prises en compte dans l'analyse.

Du fait de ces deux types de coûts externes, la quantité d'emballages (et donc de déchets) produits est supérieure à ce qu'elle serait à 1'optimum. De plus, le choix des types d'emballages est effectué sans prendre suffisamment en compte leur effet différencié sur l'environnement.

\section{L'intérêt d'une taxe sur les emballages}

La prise en compte, ou internalisation, des effets externes, nécessaire pour atteindre l'optimum, est généralement recherchée à travers trois types d'instruments (Barde, 1992, troisième partie) : les normes, les taxes et les permis négociables. Dans le domaine qui nous occupe, la dernière solution semble impossible à mettre en place ; on imagine mal en effet les ménages échanger des « droits à acheter des emballages ». Les normes peuvent être utilisées pour interdire certains matériaux considérés comme nuisibles (ainsi, certains pays comme la Suisse ont interdit les bouteilles en PVC), mais ne sauraient assurer l'internalisation des coûts externes pour les autres types d'emballages. Reste l'instauration d'une taxe qui peut porter soit sur les produits emballés, soit sur l'enlèvement des ordures de façon proportionnelle au poids rejeté par chaque ménage. Dans les deux cas, le principe pollueur-payeur serait respecté. Toutefois la seconde solution semble difficile à appliquer dans la mesure où elle inciterait les ménages à rejeter leurs déchets de façon « sauvage ».

Pour atteindre l'optimum social, la meilleure solution semble donc de mettre en place une taxe sur les futurs déchets, c'est-à-dire sur les emballages non réutilisés (9). Pour chaque type d'emballage, cette taxe devrait être égale au coût externe marginal que ce type d'emballage entraînera lorsqu'il sera devenu déchet. En pre- mière approximation, on peut considérer que pour chaque catégorie de matériau, le coût externe est proportionnel au poids. La taxe devrait donc être assise sur le poids des emballages, avec une différenciation par matériau. Un tel système est défendu en particulier par Pearce et Turner (1992). Calculer une taxe qui prenne en compte l'ensemble des coûts externes suppose de distinguer, pour chaque matériau, les différents modes de gestion (10) utilisés. Pour chaque mode de gestion, il faut ensuite estimer le coût social par unité de poids, somme du coût de la collecte et du traitement et du coût associé aux dommages pour l'environnement. La taxe serait alors calculée pour chaque matériau en pondérant le coût social de chaque mode par sa part pour le matériau en question.

La France et l'Allemagne, nous l'avons vu, ont fait des choix différents quant aux modes de gestion adoptés. En particulier, l'Allemagne s'est fixé des objectifs de recyclage beaucoup plus ambitieux. Comment juger de la pertinence de ces décisions? En théorie, il est possible de déterminer un niveau optimal de recyclage (cf. encadré 2), mais en pratique c'est extrêmement difficile ; en effet d'une part la connaissance de la courbe de coût marginal de la collecte sélective reste imparfaite, d'autre part les résultats des tentatives d'évaluation monétaire des dommages pour l'environnement sont fragiles et souvent contestés (11). Du fait de ces obstacles, les objectifs de recyclage imposés à la DSD et à Éco-Emballages sont moins l'aboutissement d'une analyse coûts-avantages que

9. Pour être tout à fait exact, précisons qu'il faudrait aussi taxer, mais à un taux inférieur, les emballages réemployés, car ceux-ci ne sont jamais réutilisés à l'infini. Ainsi, une bouteille de verre consignée qui est retournée plusieurs fois par les ménages devrait être moins taxée que la même bouteille non consignée. 10. Nous appelons ici " mode de gestion " la combinaison d'un mode de colecte et d'un mode de traitement. Par exemple : collecte par une « deuxième poubelle " suivie d'un tricentralisé puis d'un recyclage, collecte indifférenciée suivie d'une incinération, collecte par conteneur en apport volontaire suivie d'un recyclage...

11. Sur ce sujet, on pourra se reporter à l'encadré de l'article de D. Litvan dans ce numéro.

\section{Schéma III}

Le cycle de vie de l'emballage

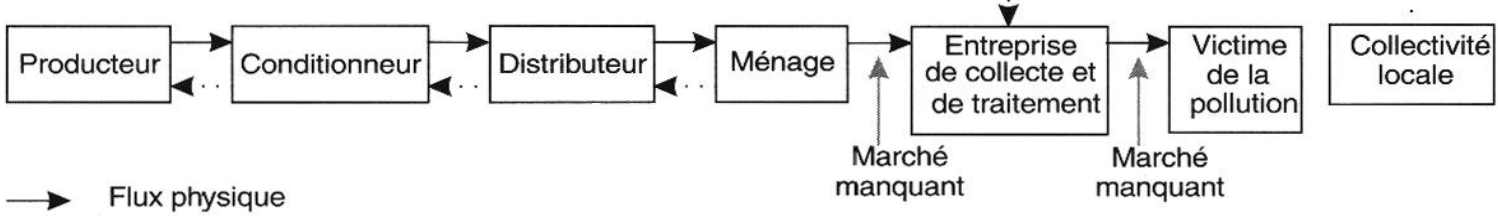

4... Flux financier 
de «paris » sur les potentialités des modes de gestion en concurrence (incinération contre recyclage en particulier).

Ces incertitudes rendent également difficile le calcul de la taxe optimale. Toutefois, il est dès aujourd'hui possible de fixer le montant de la taxe de façon approximative, puis de l'ajuster lorsque notre connaissance des coûts s'améliorera. Une telle taxe devrait entraîner trois types d'évolution :

E1 - une réduction du nombre d'emballages produits, suite à leur renchérissement ; on peut

\section{Encadré 2}

\section{LA DÉTERMINATION DU NIVEAU OPTIMAL DE RECYCLAGE}

En théorie, on détermine le niveau "optimal " de recyclage comme celui qui minimise le coût social total de la gestion des déchets. Nous présenterons ici le principe d'un arbitrage entre la collecte sélective suivie d'un recyclage d'une part, et la collecte indifférenciée suivie d'un traitement unique, par incinération ou mise en décharge, d'autre part. Un tel arbitrage nécessite de connaître les courbes de coût marginal de ces deux modes de gestion.

On estime généralement (1) que le coût de la collecte sélective est croissant lorsqu'on vise à récupérer une fraction de plus en plus importante d'un gisement donné. En même temps, la qualité et donc la valeur des déchets récupérés diminuent puisque l'exploitation du “ gisement » constitué par les déchets commence par les fractions les plus intéressantes. Les rendements d'échelle de la collecte sélective sont donc décroissants. Certes, il est possible qu'à l'inverse, le recyclage de certains matériaux se caractérise par des rendements d'échelle croissants ; globalement toutefois, il est raisonnable de retenir l'hypothèse de rendements d'échelles décroissants pour le couple collecte sélective et recyclage. À l'inverse, l'incinération et la mise en décharge bénéficient de rendements fortement croissants du fait de l'importance des coûts fixes.

Le schéma présente pour chacun des deux modes de gestion, "indifférencié " et "sélectif", la courbe de coût marginal, net des recettes de valorisation. La quantité de déchets est donnée et doit être partagée de façon optimale. L'axe des abscisses se lit de gauche à droite pour la gestion sélective et de droite à gauche pour la gestion indifférenciée.
Pour une collectivité locale qui cherche à minimiser le coût de la gestion de ses déchets, la combinaison optimale se situe au point $Q_{1}$, qui correspond à l'intersection des deux courbes de coût marginal. En effet, accroître la part de la gestion indifférenciée revient à se déplacer à gauche de ce point, où le coût marginal de ce mode est supérieur à celui de la gestion sélective, ce qui accroît le coût global ; il en est de même pour un développement de la gestion sélective à droite de $\mathrm{Q}_{1}$.

La prise en compte des dommages que le traitement par incinération ou mise en décharge cause à l'environnement déplace vers le haut la courbe de coût marginal de la gestion indifférenciée (courbe en pointillés sur le schéma) (2). L'optimum se situe alors au point $Q_{2}$, qui correspond à un coût financier plus élevé pour la collectivité locale, mais à un coût social plus faible du fait de dommages pour l'environnement inférieurs. Une intervention de l'État est alors nécessaire pour permettre un développement du recyclage au-delà du point $Q_{1}$, c'est-à-dire au-delà de ce qui est rentable pour les collectivités locales.

1. Ces hypothèses, ainsi que le principe du schéma, sont empruntés à Bertolini, 1987.

2. Pour simplifier, on néglige dans ce raisonnement les dommages que la gestion sélective peut causer à l'environnement. Leur prise en compte conduirait au même type de résultat si l'on considère qu'ils sont inférieurs aux dommages causés par l'incinération ou la mise en décharge.

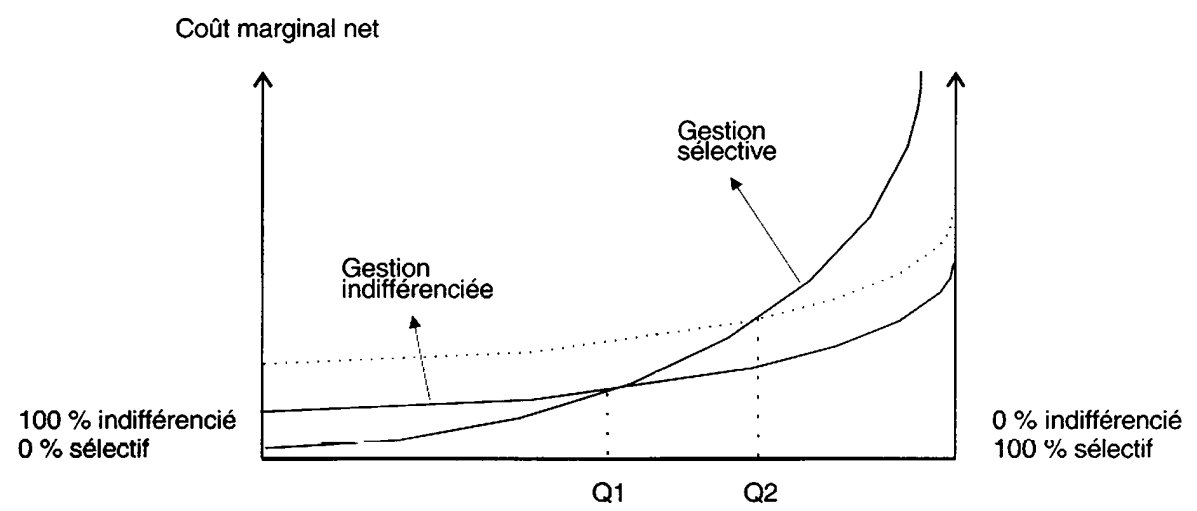


penser en particulier à la suppression de certains suremballages ainsi qu'au développement de la réutilisation, en particulier par la consignation ;

E2 - un développement relatif des emballages les plus légers vis-à-vis des emballages les plus lourds pour un même matériau, puisque la taxe est proportionnelle au poids ;

E3 - des substitutions entre matériaux, du fait de la variation des prix relatifs.

Dans quelle mesure les systèmes français et allemand respectent-ils les préceptes de la théorie économique?

\section{Éco-Emballages et le principe pollueur-payeur : trois remarques}

À première vue, la contribution payée à ÉcoEmballages par les conditionneurs se rapproche de la taxe sur produit que nous venons d'étudier, mais le mode de calcul de la contribution ne répond pas à un objectif d'internalisation des externalités. En effet, la contribution a été créée pour financer le développement du recyclage : celui-ci suppose un développement massif de la collecte sélective et du tri des déchets, ce qui entraîne un surcoût par rapport à la collecte indifférenciée. Le niveau de la contribution ( 3 centimes par emballage à terme) a été calculé de façon à couvrir ce surcoût, c'est-à-dire la différence entre le coût de la collecte sélective et du tri (net du produit de la vente des déchets triés), estimé à partir de l'expérience de Dunkerque (12) et le coût de la collecte indifférenciée suivie d'une incinération aux normes avec récupération d'énergie (13) (cf. schéma IV).

Si l'on compare la contribution Éco-Emballages à la taxe optimale proposée par la théorie, trois remarques peuvent être formulées.

- Le taux de la contribution est faible.

Le dispositif n'impute au consommateur qu'une fraction du coût que l'emballage entraînera pour la collectivité locale lorsqu'il sera devenu déchet (le surcoût de la collecte sélective et du tri) et non l'ensemble de ce coût. La partie restante devra être financée par le contribuable. De plus, les dommages pour l'environnement causés par les déchets d'emballages ne sont pas pris en compte.

- La contribution n'est pas assise sur le poids de l'emballage.
- Elle n'est pas différenciée par matériau.

Or, le coût externe associé à chaque type d'emballage est approximativement proportionnel au poids et varie selon le matériau utilisé. Pour fournir les bonnes incitations, les prix relatifs des matériaux devraient refléter ces différences.

La contribution d'Éco-Emballages apparaît très éloignée d'une taxe «pollueur-payeur » optimale. Peut-on malgré tout s'attendre à des évolutions favorables? Les substitutions entre matériaux (E3) semblent exclues, puisque la contribution n'est pas différenciée. De même, un développement des emballages les plus légers (E2) n'a aucune raison de se produire, dans la mesure où la contribution est calculée à 1'unité et non au poids.

S'agissant de la réduction de la quantité d'emballages produits (E1), elle ne peut survenir que si la contribution atteint un niveau suffisant ; mais avec un taux de 1 centime par emballage, taux actuel, ou même de 3 centimes, taux prévu à terme, on ne saurait s'attendre à un réel effet incitatif. Toutefois, indépendamment des incitations monétaires, il se peut que l'existence des dispositifs de collecte sélective accélère la prise de conscience par les ménages des problèmes que pose le traitement des déchets. D'une façon un peu détournée, on pourrait alors voir les consommateurs éviter certains suremballages qui n'ont bien souvent qu'un rôle « mercatique » et assister à une diminution du nombre d'emballages produit.

\section{Schéma IV \\ Décomposition des coûts monétaires} liés aux déchets*

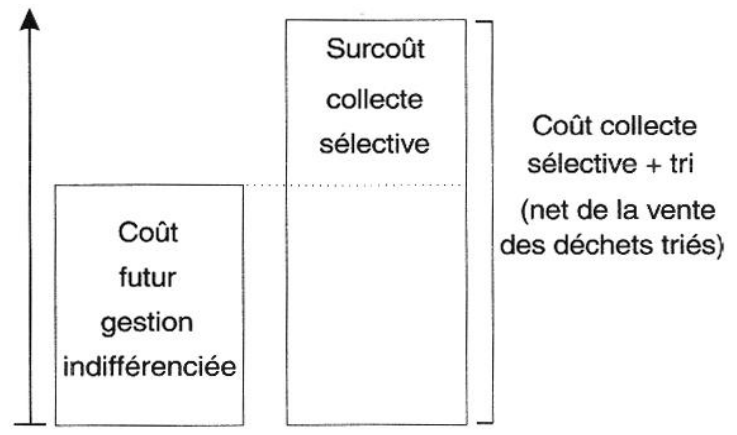

* Schéma inspiré par Y. Martin. 


\section{La DSD et le principe pollueur-payeur : une mise en conformité partielle}

Depuis octobre 1993, la taxe perçue par la DSD est calculée au poids et par matériau, sur la base des coûts de collecte et de tri des déchets. De ce fait, elle joue à la fois un rôle de financement, comme la contribution française, et un rôle d'internalisation, dans la mesure où le prix des produits emballés intègre ainsi le coût de la gestion de leurs déchets d'emballages. Ce barème, dont les principes ontété repris et affinés dans la version d'octobre 1994, a explicitement pour but d'inciter les acteurs économiques à réduire la quantité d'emballages et à choisir les matériaux les plus facilement recyclables. En revanche, les dommages pour l'environnement ne sont pas internalisés. Selon une analyse effectuée par le bureau d'études allemand GVM, il semble que l'ordonnance Töpfer ait commencé à produire les effets prévus par la théorie. Entre 1991 et 1993, la production d'emballages est passée de 12,8 à 11,8 millions de tonnes (cf. graphique) soit un renversement de tendance très significatif par rapport aux hausses des années précédentes, ce qui peut correspondre aux évolutions E1 et/ou E2 de notre typologie (14). De même, des effets de substitution entre matériaux commencent à apparaître (évolution E3). La part des emballages complexes et des matières plastiques diminue (avec une baisse des plastiques de 922000 tonnes en 1991 à 850000 en 1994) au profit du verre et du papier-carton (DSD, 1995).

\section{En France : une mise en œuvre progressive}

L'analyse comparative menée dans cet article aura permis d'avancer dans trois directions. En premier

\section{Graphique}

\section{Évolution de la consommation totale}

En millions de tonnes

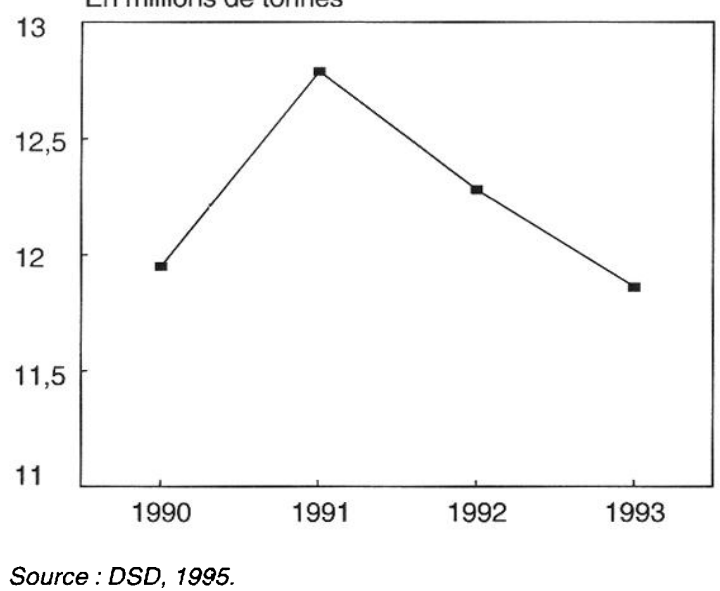

lieu nous avons vu que, en n'ayant aucune influence a priori sur la production d'emballages, le système français de gestion des déchets d'emballages ménagers est loin de permettre l'internalisation des effets externes, alors que la politique allemande semble au moins partiellement guidée par ce principe. En second lieu, la mise en place de la politique française relève d'une « gestion du temps » (15) beaucoup plus prudente, et sans doute plus réaliste, qu'en Allemagne. En effet, le délai fixé à la DSD pour atteindre ses objectifs (juillet 1995) s'est avéré insuffisant pour que les capacités de recyclage des matières plastiques s'adaptent à l'afflux de déchets à traiter. En troisième lieu, la politique française laisse une grande liberté de manœuvre aux opérateurs décentralisés quant aux choix des filières techniques alors que l'Allemagne a délibérément restreint ce choix en interdisant l'incinération des emballages. Cette ouverture de l'horizon des techniques possède plusieurs avantages : minimisation du coût moyen à court terme, incorporation rapide des innovations techniques, définition de graphiques d'organisation collant au plus près aux demandes et aux besoins des collectivités locales (Defeuilley et Godard, 1994).

Néanmoins, le « décollage » de la collecte sélective en France ne se fera que si la volonté politique, présente à l'origine, subsiste. Un scénario «noir » est en effet facile à imaginer. Si les fabricants de matériaux sont intransigcants sur les conditions de reprise des déchets triés, en termes de qualité, les collectivités locales rechigneront à s'engager dans la collecte sélective.

Les analyses présentées montrent deux politiques qui se fondent sur des compromis institutionnels et sur des « paris » technologiques très contrastés. Les résultats disponibles étant très partiels, une évaluation ultérieure sera nécessaire pour en apprécier les conséquences à long terme.

14. Cette diminution de la consommation d'emballages n'est pas imputable à l'évolution générale de l'activité économique, puisque sur la même période, le PIB a augmenté de $1 \%$ en termes réels.

15. Godard (1994) a mis l'accent surl'importance de la " gestion du temps " dans les problèmes d'environnement. Ces derniers sont en effet caractérisés par une double menace d'irréversibilité, celle des changements dans l'évolution biophysique et celle qui résulte de la fermeture d'options technologiques et économiques. Dès lors, les décideurs doivent prendre en compte les différents rythmes qui animent l'environnement, comme les variables socio-économiques, en cherchant à éviter les deux écueils que sont une réaction trop tardive et une fixation prématurée de règles bloquant l'innovation. 


\section{BIBLIOGRAPHIE}

Barde J.-P. (1992), Économie et politique de l'environnement, Paris, PUF.

Beffa J.-L. (1991), Rapport sur la valorisation des déchets d'emballages domestiques, rapport au ministère de l'Environnement.

Bertolini G. (1987), « Économie de la collecte des résidus ménagers : les articulations entre récupération et élimination », Revue d'Économie politique, vol. 97 , $n^{\circ}$ 5, pp. 631-648.

Bertolini G. (1992), «Les déchets, rebuts ou ressources ? , Économie et Statistique, $\mathrm{n}^{\circ}$ 258-259, pp. 129-134.

Bially E. (1993), Le système dual allemand - Ce qu'il est en réalité, Dual System Deutschland (DSD), Francfort.

Defeuilley C. et Godard O. (1994), Regards économiques sur la nouvelle organisation de la gestion des déchets ménagers en France, Cired, Paris.

DSD (1994), Werkstoffrecycling in zahlen, Duales System Deutschland, Francfort.

DSD (1995), The New Scale of Fees for the Green Dot, Duales System Deutschland, Francfort.

Éco-Emballages (1992), Demande d'agrément, I evalloisPerret.
Éco-Emballages (1993),Éco-Emballages et les collectivités locales, Levallois-Perret.

Glachant M. (1995), « Les accords volontaires dans la politique environnementale : une mise en perspective de leur nature et de leur efficacité », Économie et Prévision, $\mathrm{n}^{\circ} 117-118$, pp. 49-59.

Godard O. (1994), « Stratégies industrielles et conventions d'environnement : de l'univers stabilisé aux univers controversés », Insee Méthodes, $n^{\circ}$ 39-40.

OCDE (1992), « Réduction et recyclage des déchets d'emballages ", Monographie sur l'environnement, $n^{\circ} 62$.

Pearce D.W. et Turner R.K. (1992), « Packaging Waste and the Polluter Pays Principle. A Taxation Solution », CSERGE Discussion Paper, $\mathrm{n}^{\circ}$ 92-01.

Quirion P. (1994), « La gestion des déchets d'emballages ménagers en France et en Allemagne : éléments d'évaluation économique ", mémoire, DEA "Économie de l'environnement et des ressources naturelles", université de Paris-I.

Riboud A. (1991), Emballages et environnement, rapport au ministère de l'Environnement. 